\title{
The rhetoric of computer simulations in astrophysics: a case study
}

\author{
Aimee Kendall Roundtree
}

\begin{abstract}
This article is a case study and rhetorical analysis of a specific scientific paper on a computer simulation in astrophysics, an advanced and often highly theoretical science. Findings reveal that rhetorical decisions play as important a role in creating a convincing simulation as does sound evidence. Rhetorical analysis was used to interpret the data gathered in this case study. Rhetorical analysis calls for close reading of primary materials to identify classical rhetorical figures and devices of argumentation and explain how these devices factor in the production of scientific knowledge. This article describes how abduction, dilemma, compensatio, aetiologia, and other tactics of argumentation are necessary in creating the simulation of a supernova. Ultimately, the article argues that rhetorical mechanisms may be responsible for making some simulations better and more sound than others.
\end{abstract}

\section{Introduction}

This article is a case study and rhetorical analysis of the drafting process of a specific research paper on a computer simulation in astrophysics, an advanced and often highly theoretical science. The term "computer simulation" refers to computer programs (and resulting illustrations and visuals) that use algorithms and other advanced programming code to predict how otherwise unknown phenomena occur or behave in nature. Computer simulations are products of high-level mathematical equations meant to model phenomena in behavior and appearance. They are often used to predict or explain complex systems or to replace first-hand observation in theoretical sciences such as forensics, palaeontology, astrophysics and others. Examples include simulations that NASA used to determine what happened to cause both the Challenger and Columbia accidents, the models that meteorologists use to forecast what storm fronts and other weather patterns mean for the weather in your local area, and the predictive models that climatologists use to help explain how pollutants will affect the environment and global climate in the future.

Broadly speaking, simulations have important value as evidence in scientific investigation particularly in scientific fields where direct observation of phenomena is difficult or impossibile because of their capacity for verisimilitude. In a prior article, I discuss how simulations are more persuasive than thought experiments, metaphor and simple models, because simulations are virtually what they represent:

\begin{abstract}
Simulations yield virtual results...[For example, a child] can virtually have had signs of the measles both before and after we know whether or not he actually has the virus...particularly if the spots on his skin prevented him from going to school, left longstanding scars or caused any other measleslike results... [Virtual] data needn't be conclusive or absolute to lend meaning. That it contains the virtue of a thing--the worth and workings of it--gives it capacity to explain the thing itself. ${ }^{1}$
\end{abstract}

In this way, simulations go a step beyond thought experiments, metaphors and other modes of scientific invention, all of which being forms of what Aristotle calls mimesis (e.g., imitation or role playing). ${ }^{2}$ A simulation not only resembles the thing it represents, but it also stands in for it or produces it before our very eyes, which is more akin to Aristotle's notion of energeia or "bringing before the eyes" through a process of weighing conditions and balancing contraries. ${ }^{3}$

In this article, I will show how sound rhetorical decisions enable the weighing of conditions that goes into creating a convincing simulation. Winsberg describes the process of making simulations as ad hoc 
(e.g., based on what is improvised and impromtu rather strategic and calculated). Unlike typical computer programs which proceed solely from binary equations, simulations require a little ingenuity on the programmer's part. Winsberg describes the process:

Simulationists use ad hoc modelling assumptions to help make their computational models more tractable and manageable. Ad hoc modelling assumptions include such techniques as simplifying assumptions, removal of degrees of freedom, and even substitution of simpler empirical relationships for more complex but also more theoretically founded laws. ${ }^{4}$

When creating a simulation, programmers have to make assumptions about the systems they are recreating, make substitutions for elements or components difficult or impossible to calculate, and follow hunches rather than observations about the objects they are representing. Winsberg identifies specific ad hoc decisions that simulationists face. ${ }^{5}$ They have to eliminate data from their computer models; they have to invent elements especially for their model design; they have to choose initial conditions from which their models will develop; and they have to set the parameters of their models Since simulations are used to study systems and phenomena that are difficult if not impossible to study first-hand, they often cannot base these decisions on observed evidence.

These ad hoc decisions have a degree of subjectivity, so they invite errors above and beyond those created by improper programming language syntax. Simulationists commit eliminative errors by neglecting to include what their peers agree are significant features of a system. ${ }^{6}$ They make creative errors by adding artificial elements that their peers believe should not substitute eliminated ones. ${ }^{7}$ Finally, they can perpetrate errors when setting the parameters of the simulation. For example, they must set the increments and initial values from which the action of the simulation progresses from time step to time step. Setting improper initial conditions could yield contrived or particular results. ${ }^{8}$

Winsberg calls this ad hoc component of the simulation processes "fudging":

Sometimes simulationists will ignore factors or influences, not because they are deemed 'external,' but because limitations of computational power make their inclusion impractical. That is what I refer to as eliminative ad hoc modelling. In this case, the simulationist has one of two options: either to assume that the effects of this omitted factor are negligible or to make use of some sort of empirical 'fudge factor' (creative ad hoc modelling), to make up for the absence of neglected factor.

However, to call these decisions ad hoc or arbitrary overlooks how the process involves careful, purposeful reasoning and explanation of motivations and intentions to colleagues within the scientific community. Even the correctives for these errors suggest that ad hoc modelling is not arbitrary. For example, Winsberg recommends that simulationsists use pre-existing simulations as a guide, and that they run multiple versions of the simulation, each incorporating different ad hoc decisions than before. ${ }^{10}$ In this way, ad hoc modelling involves rhetorical choice insofar as it calls for reasoned deliberations, awareness of audience, and purposeful selection of supporting evidence.

Rhetorical choice is so integral to simulation programming that a failure of rhetoric can cast doubt on the findings that the simulation produces. For example, concerns about climate change simulations are a part of what fuelled the "Climategate" controversy in November 2009, when emails and documents from the University of East Anglia's Climatic Research Unit (CRU) — one of the world's leading research facilities that publishes data proving global warming - were leaked on the Internet. The documents that climate sceptics found most troubling were emails wherein CRU researchers call one of their algorithms a "trick" used to "hide the decline" in modern temperature amidst an overall temperature increase. ${ }^{11}$ Climate skeptics heralded this language as proof of misdeed on the part of the CRU team. However, independent investigations found that those seemingly deceitful terms were "colloquialisms" for orthodox statistical and computer programming methods commonly used to reconcile disparate data sets. ${ }^{12,13}$ My article will demonstrate how these kinds of deliberations are not disingenuous or purely semantic, but rhetorical and necessary for computer simulation methodology.

This article will detail the rhetorical deliberation undertaken and strategies employed to create a supernova simulation. First, this article describes the methods of my rhetorical analysis, then it will discuss specific rhetorical strategies used in the simulation article drafts and code. Finally, it will describe the implications of the argumentation that underpins computer simulations. 


\section{Case Background}

This article will examine a journal article about a simulation project performed by astrophysicists and computer scientists at the Oak Ridge National Lab (ORNL) in Tennessee. The Terascale Supernova Initiative (TSI) team investigates the mechanisms behind supernovae explosions. Supernovae occur in the final stage of a star's evolution. A star begins as a protostar (clouds of gas and dust). Gasses and other elements pulsate through collapsing stars. Gravity pulls the floating particles together and heats up the cluster (a.k.a. accretion), but not at uniform rates or pressures. ${ }^{14}$ The inconsistency precipitates an explosion that has the potential to synthesize chemical elements in the Periodic Table.

The assumption has been that these waves dissipate and energy dwindles as a star dies. Therefore, some other force or element must provoke the huge explosion. Neutrinos (neutral subatomic particles) within the star's core seemed the most likely culprit. ${ }^{14}$ The TSI team's groundbreaking work, however, reveals that small gaseous perturbations (accretion shocks) in the star's core can accelerate the waves and possibly create enough energy to fuel the explosion. Their model proposes an alternative means besides neutrinos for the supernova to generate energy.

The TSI team ultimately published their work - entitled "Stability of Standing Accretion Shocks with an Eye Toward Core Collapse Supernovae" (which I'll call the SAS paper, article, draft and simulation from this point on) - in the Astrophysical Journal in 2003. ${ }^{15}$ John Blondin (Department of Physics, North Carolina State University, Raleigh, NC) was the lead author, and Anthony Mezzacappa (PhysicsDivision, Oak Ridge National Laboratory, Oak Ridge, TN) and Christine De Marino (Department of Physics, North Carolina State University, Raleigh, NC) coauthored the article. This rhetorical analysis examines the publication, preceding drafts, computer code and correspondence from the authors to each other and to journal reviewers, all recorded in email transmissions.

Theoretical sciences often depend upon simulations rather than laboratory experiments as primary evidence to explain phenomena. The astrophysics article under examination in this study proposes a new mechanism for understanding how supernovae explode. The case study is also important insofar as it helps to situates ad hoc modelling as a legitimate mode of rhetoric and argumentation.

\section{Methods}

In order to track the rhetorical underpinnings of the SAS article, I reviewed multiple prior drafts, the published article, email correspondence, and some of the computer program used to help create the simulation itself. I requested and received the primary sources from Blondin and Mezzacappa. I compared two different versions of the computer code partially responsible for creating the simulation one available online since 1991 and the other since 1998. I analyzed the differences between how the two versions handled comments within the code and semantic cues. Comments are natural-language guideposts that programmers write within code to explain a sub-routine's function; computer scientists have yet to reach consensus on when and how to use them. The Human Subjects and Institutional Review Board at the University of Texas at Austin reviewed the study and deemed it exempt on the grounds that it was a study of existing data and documents. The complete case study also included phone conversations with the principal investigators, analyses of secondary sources (including conference presentations, news clippings, government reports and other sources that cited the project). However, this article presents findings from the most central and important primary, written materials.

I use rhetorical analysis to examine the drafts. Jeanne Fahnestock, Leah Ceccarelli and others have used similar analyses, which call for close reading of primary materials to identify classical rhetorical figures and devices of argumentation in expository writing and speech. ${ }^{16,17}$ They have argued that figures of speech are essential in science for explaining key scientific concepts and theories. In this way, rhetoric serves not only as a means by which to persuade the scientific community to support one theory over another, but also as a foundational tool in the production of scientific knowledge itself.

\section{Results}

Upon what does the SAS team base their simulation? The team enlists physical evidence and rhetorical acumen. This section will describe some of the most important and prominent features of the drafts and code. 
Physical evidence. In the SAS simulation and article, Blondin, Mezzacappa and DeMarino use whatever physical evidence possible to construct and report its supernova model. Supernovae happen at extreme heats, pressures and distances; they are inaccessible, save for residual evidence at micro- and macroscopic levels. In spite of these odds, even astrophysics isn't altogether without physical evidence, albeit indirect. For example, scientists look for evidence that reaches our atmosphere from even farexploding supernovae. Supernovae emit photons across the optic spectrum, and telescopes can measure gamma rays, x-rays and the like. Supernovae also emit neutrinos which reach this atmosphere. Neutrino detectors can yield some information about supernovae. To be fair, the evidence is scarce. To put the scale of the information into perspective, consider the fact that the Kamiokande - the neutrino detector cited in the work awarded the Nobel Prize in Physics in 2002 - captured little over a dozen of the estimated trillions of neutrinos emitted from the supernova in question. ${ }^{18}$

For example, in this case, Blondin, Mezzacappa and DeMarino base the behavior of an unknown (the waves of gas in the supernova) upon what is already known from laboratory physics about the behavior of wave particles - namely, how waves of gas behave under certain levels of pressure, heat and rotation. The team cites physical evidence throughout the published manuscript and in their response to reviewers. They include the following in an email to the journal reviewers: "We presented Figure 7 to illustrate the feedback between vorticity generated at the shock and pressure waves generated from the turbulent flow at small radii fed by the vorticity." Vorticity is a foundational concept in fluid dynamics. Wave behaviors have been tested and well-established with laboratory experiments. Blondin, Mezzacappa and DeMarino use observable evidences to help support their claims about an unobservable event - namely, supernova mechanisms.

How the authors use physical evidence here falls in line with prior work in the rhetoric of science about the rhetorical nature of physical evidence and scientific facts. Latour and Woolgar discuss how scientific facts have meaning through socially constructed networks or sets of positions in a discipline. ${ }^{19}$ Also, Danette Paul describes how scientists cite facts from prior research for specific rhetorical ends - for reinforcing their level of certainty and for creating a research space upon which their audience can agree and from which they can judge their claims. ${ }^{20}$ In this case, the SAS team uses well-established facts from physics to scaffold their new claims about an alternative mechanism for supernovae explosions. They cite widely accepted concepts and citations to explain and justify the new mechanism. While these facts in and of themselves are insufficient to prove the new mechanism, and while obtaining more physical evidence and direct observation of supernova explosions is impossible with current technology, using commonly shared sets of positions does help make their case more convincing.

Abduction. TSI's conclusions develop via abduction. Peirce describes abduction as an alternative to induction and deduction in science. "Abduction is an argument which presents facts in its Premiss which present a similarity to the fact stated in the Conclusion, but which could perfectly well be true without the latter being so, much more without its being recognized. ${ }^{21}$ Induction, deduction, and abduction would treat the same premises differently. Take, for example, the different conclusions you can draw from different combinations of similar facts. Deduction takes what is true of the general case and applies it to a specific case: All humans die (general, major premise). Socrates died (minor premise), then he was human (specific conclusion). Induction generalizes facts from the specific case: Socrates was human (specific, major premise) and he died (minor premise). So, all humans must die (general conclusion). Abduction interchanges the conclusion for what would be considered a premise of an inductive or deductive syllogism: All humans die (general, major claim); Socrates was human (specific, minor claim), so he died (conclusion).

In deduction, the strength of one or the other premise warrants making the leap to the conclusion. If the general premise is true, then it strengthens the specific conclusion. Induction and abduction yield problematic warrants - warrants that, from a symbolic logical perspective, don't necessarily strengthen if their premises are tautologies. Abductive statements are not necessarily true; they don't have the logical form that resists falsehood. For example, the team uses abduction to brainstorm a major conclusion. Emails between the authors included the following:

If the explosion is indeed 'jet-like'..., what would the ejecta density distribution look like at late times -- i.e., hundreds of years later as in Cas A?

Here, a general premise (explosions are "jet-like") and specific premise (what ejecta might look like in cases like Cas A) are linked together. However, unlike in induction or deduction, in the case of abduction, neither the general nor specific premises have been proven beyond the shadow of a doubt. And, these contingent premises are used to make hypothetical conclusions. 
Rhetorical figures. In addition to abduction, other rhetorical strategies and arguments underpin the decision making process that produces simulations. In the first draft, for example, Blondin, Mezzacappa and DeMarino set up a dilemma or double propositions wherein either they set a particular initial boundary, or they risk degrading their results. ${ }^{22}$ In the excerpt that follows the team explains how set boundaries that constrain their simulated supernova. Since a part of testing a supernova involves measuring how much energy escapes out of the system, they must set a leaky boundary out from which the energy can flow. The excerpt below explains why they made the leaky boundary as they did, despite the fact that it created possible "noise" or errors inherent to the boundary equations themselves - my bold for emphasis.

The pressure and density at this leaky boundary are adjusted to force zero gradients...

(We could also have equated the pressure gradient and the gravitational acceleration, but the cancellation involved would most likely have proven too noisy for our purposes.)

In this dilemma, the team chose one equation over another for setting values for the gas flowing out of the system. Had the team not set an initial condition, more dire consequences would ensue. Later drafts presented the decision to set the boundary affirmatively — as "influenced by the desire to construct timedependent solutions" rather than to avoid complications such as noisiness.

Another passage in the second draft described why the team used a problematic algorithm. The point of the paragraph was to admit the drawback - namely, that it, too, caused noise in the system calculations, noise that might have influenced or tainted their results. "We find that this problem is worse in three dimensions than in two." The sentence was meant to offset the problematic algorithm vis-à-vis compensatio - weighing or counter-balancing one distasteful extreme with another. ${ }^{23}$ When computer simulations reconstruct a complex system, they can do so in one-, two- or three-dimensions. Systems that look and behave the same all over translate into one dimension. Systems that have two identical sides require two-dimensions. Systems with heterogeneous, dissimilar parts require three dimensions. ${ }^{24}$ The SAS simulation required two- and three-dimensional runs. With compensatio, even if the problem occurred in two dimensions, the problem wasn't nearly as bad in three dimensions, where TSI used it.

Next, the first draft contained moments wherein the team justifies the simulation's initial conditions. For example, in a sentence included in the first draft (but absent from the second), the team explained that they must impose a leaky boundary (e.g., the end point through which gas and energy escapes) as an initial condition for the simulation. "In this way, we ensure that perturbations to the setting solution, which would dominate this solution as we approached the origin, are advected off the grid." The team wanted to test if the standing accretion shock is what causes the star's energy to build up, so they had to eliminate any other factors that might affect the system's energy. They added the leaky boundary so that any other system disturbances would move off into the horizon (or advect) rather than feed or starve their simulated supernova. This sentence in the earlier draft explained the need for the leaky boundary - a rhetorical move called aetiologia, wherein writers enumerate cause or reasons. ${ }^{25}$

Further, the first draft contained statements justifying some of the creative additions that the team made. In constructing the system's boundaries, they had to set constant accretion rate (e.g., the rate at which gasses and particles accumulate or build). The decision is a creative one, because it runs contrary to the accepted notion that core collapse supernova have decreasing accretion rates. In a parenthetical statement in the first draft - my bold for emphasis - Blondin, Mezzacappa and DeMarino attempt to foresee and redress their audience's reservations about their decision:

The fluid variables at the outer boundary are held fixed at values appropriate for highly supersonic free-fall at a constant mass accretion rate. (While this does not reflect the decreasing accretion rate in a core collapse supernova,... a constant mass accretion rate is imposed to obtain an analytic solution. Nonetheless, this should not affect the conclusions drawn here.)

Here, Blondin, Mezzacappa and DeMarino admitted that they lose one virtue (a decreasing accretion rate that resembles more an actual core collapse supernova) to gain another (an analytic solution). They also assure the reader that this difference (between the supernova's actual accretion rate and the one they model here) will not affect their conclusions about whether accretion shocks do, indeed, cause an instability that helps stars explode. The move typifies concessio, or conceding a point to prepare for a more important one. ${ }^{26}$

The earlier drafts reveal more of the decisions and choices that governed the team's work. These earlier drafts reveal some of the rhetorical strategies — dilemma, compensatio, aetiologia, concessio — that went into making the ad hoc decisions. The team makes reasoned arguments to decide their boundary conditions and make creative additions. 
Citations and narrative in code comments. Novel features of the computer program code itself also aids in building a convincing argument. Blondin used FORTRAN to develop Virginia Hydrodynamics \#1 (VH1), the software an adaptation of which the TSI team used for the simulated supernova in question. A few studies and stylebooks recommend and analyze typical comment format. Usually comments are written in imperative or indicative mode to indicate to programmers the purpose of the sub-routine. ${ }^{27}$ They also serve different functions. Some define sub-routine content or elements (definitional), some describe what the sub-routine produces (descriptive), some give programmers instructions about subroutines to add or other maintenance to perform (instructional), and others explain how a sub-routine works (operational). ${ }^{28}$

Although most comments usually define or detail the function of the subroutines they precede, comments in VH1 had unorthodox traits. For example, the comments contained in-text citations to others' work. I found that both old and new versions shared 3 sub-routines - named parabola.f, riemann.f, and paraset.f — with comments that included in-text citations to the articles that inspired the underlying architecture. Here is a sample comment from the sub-routine parabola.f: "Colella and Woodward, JCompPhys 54, 174-201 (1984) eq 1.5-1.8,1.10 // parabola calculates the parabolas themselves. call paraset first for a given grid-spacing to set the constants, which can be reused each time parabola is called." In this case, the team frames operational and descriptive comments with a citation from the Journal of Computational Physics. The citation also serves to show the reader that the programmers had backing from two others in the scientific community. Here, the VH1 program also invites readers and users to see for themselves how the original programmers derived the routine. The reader already knows that the programmers had a hand in composing the sub-routine. Now, the reader can also identify the very article or book upon which the sub-routine is based. Including citations implies that the code is a product of research. Citations in the comments also go to show that scientists often borrow ad hoc decisions and other features of others' simulations. Others' simulations stand as a basis for justifying using particular algorithms and imposing initial parameters.

Narrative also factors prominently in the VH1 comments. If you recall, narrative was not among the discursive modes that typify comments (e.g., description, definition, operational, instructional). ${ }^{27}$ Almost all of the new sub-routines that set initial conditions (init_bow, init_hzs, init khi, init_sod, init_sss, init_stb, init_swb and init_f) describe the phenomena replicated by the code, but with a difference. A typical comment might read as follows: "This routine parses the data" ${ }^{27}$ However, VH1 includes multiple narrative comments: "A supersonic wind originates at the surface, driving a bubble into the surrounding medium. 2$D$ spherical (R-theta)." This comment (and the others like it) describes the action of the supernova rather than the sub-routine. Taken together, they qualify as a narrative about the phenomena that the subroutines are meant to recreate. These unconventional features of the comments help make the simulation code more transparent than traditional code, which often only explains what sub-routines do, not how they do it or to what end. The audience of the VH1 software - the users who'll make their own simulations with the software - are invited to compare the code to the sources. They are invited to visualize what the subroutines create (rather than just what number crunching they do to the data).

\section{Conclusion}

Rhetorical figures underpin the ad hoc decisions made by computer simulationists. The TSI team made concessions, identified dilemmas, used abduction and other strategies to bring the supernova mechanism before our eyes. TSI weighed alternative findings and options in creating the supernova simulation. Tactics of rhetoric and argumentation are necessary in creating the virtual representation of actual objects. Recognizing and studying the rhetorical underpinnings of simulation production also can help make inroads into formulating a theory of reception that specifies how simulations are interpreted, appropriated, used, misused, proven and disproven.

The published manuscript did not include many of the rhetorical figures used to justify the ad hoc decisions, so readers did not have as much access to these important insights into the simulation design. However, published scientific articles rarely report much deliberation. Many scholars have shown how published articles do not represent all aspects of scientific knowledge production and discovery. ${ }^{29-31}$ Randy Harris says so emphatically: 
An experimental report... is a fairy tale that masks more of the experiment than it reports. Once upon a time, it says, there was a problem. Then, there was a method, under whose guidance we tinkered with the world. Then we got an answer, and the problem went away. ${ }^{32}$

Articles reporting scientific experiments never tell every twist and turn of the goings-on in laboratories or out in the field. Scientific articles rarely give the fullest accounting. Indeed, the linear narrative that scientists construct in articles often excludes missteps, tangential details, fruitless deliberations and other occurrences that directly and indirectly help scientists make decisions and draw conclusions. ${ }^{19,33}$ The SAS paper is no exception; the TSI team considered others' work, other alternatives, and hypothetical scenarios to make ad hoc decisions. By examining earlier drafts of the article they published about the simulation, we get to see possible rhetorical figures that the team used, beyond those in the formal report.

As is typical of science writing, the drafts evolved to background the authors' deliberations and foreground the simulation phenomena. ${ }^{34-37}$ In time, they gradually emphasized how the simulation itself necessitated setting certain parameters and initial conditions. However, the earlier rhetorical figures depicted the team deliberating between options. Many of the justifications for using one option over another happened by eliminating less attractive or (from their perspective) less desireable alternatives. Even when observational data are incomplete, sound and reasonable assumptions must underpin ad hoc decision-making. In the SAS case, their simulation of an actual supernova might seem arbitrary because they cannot compare it to a real simulation, but it is not arbitrary because good reasons support their decision-making. It may be advisable for peer-reviewed journals to change author's guidelines and editorial standards for scientific articles that report simulations. By allowing for longer word and page limits and recommending more explanation when reporting the rhetorical choices behind the simulation, journal editors might foster better understanding among colleagues in the scientific community and help prevent misunderstandings like Climategate from escalating.

The SAS case demonstrates how the rhetorical practices and genres of the scientific community play a significant part in effacing or erasing any traces of rhetorical production, a production trail that exposes the extent to which simulations do not and cannot perfectly recreate actual events. That the SAS article did not disclose more about the deliberation that went into it (the deliberation that clearly makes the simulation a rhetorical artifact) was not the fault of the computer simulation itself so much as what the scientific community has come to expect (and make) of the genre of journal articles, the process of manuscript review, and the practice of citation and reference. The SAS paper evolved to contain fewer rhetorical figures most likely due to the strictures of the scientific ethos - its compulsion to present itself as objective rather than subjective, definitive rather than experimental.

The case of the SAS simulation illustrates some of the rhetorical mechanisms responsible for making some simulations better than others. It appears that a simulation must not only be based on sound data, but also sound reasoning, argumentation and a convincing story. Newer theories demonstrate how computer technology manifests ideological and hegemonic tendencies. Galloway, for example, demonstrated the hegemonic nature of the protocols and equations that govern exchanges in information technology. ${ }^{38}$ Protocols impose a tyranny of their own insofar as they impose rules and constraints that delimit how we can transfer data and to what networks we are privy. Similarly, the equations and values that govern simulations reflect deliberate decisions made from weighing options, information from past research, choices about which options are the lesser of two evils, and so on. And generic constraints and pre-established roles within the scientific discursive community delimit how news of the simulation is reported, received, and recycled.

Winsberg describes the difficulties that simulationists currently face determining the validity and verifiability of their simulations. When it comes to simulations, verification (e.g., checking that simulations correctly represent the target system) and validation (e.g., checking that the model produces accurate numbers) are more closely intertwined than for small-scale models of simple systems. "What simulationists are forced to do is to focus...on establishing the combined effect of the models they begin with, and the computational methods they employ, provide results that are reliable enough for the purposes to which they intend to put them." ${ }^{39}$ For example, global climate is too complex to produce a computer model that perfectly matches the theories about climate change and the actual climate itself. "We will have to try and show, instead, that the combined effect of our choice of model and the computational methods produce predictions that are good enough for our policy-making needs. ${ }^{\prime 39}$ But by what measure should we determine which predictions are "good" and "reliable" enough? Rhetoric affords us the tools necessary for negotiating and evaluating premises and arguments in politics, business, advertising and other realms; I contend that it 
can do so in ad hoc modelling, as well. It is rhetorical strategy that helps persuade an audience that our choice of methods, decision to include or exclude concepts, and other deliberations are sound.

The field of computational science could benefit from more rhetorical analyses of its methods. Rhetorical education and a more transparent reporting of rhetorical methods employed could serve to illuminate and improve the science of creating computer simulation. Unfortunately, many introductory and advanced sections of simulation courses do not contain units wherein the class discusses ad hoc or rhetorical skills necessary for building simulations. ${ }^{40,41}$ Some have found that rhetoric improves how instructors teach programming and write active-learning-oriented assignments. ${ }^{42}$ I contend that the necessity of rhetorical know-how does not end for college-level computer programmers, nor does it solely train them for dealing with actual clients. It does not merely make assignments more contextualized, interesting and engaging. Rhetorical strategies help rationalize and ground the ad hoc reasoning necessary for creating simulation programs.

\section{Notes and references}

1 A. Kendall (2005), Computer Simulations in Computational Science: Mimesis of a Virtual Nature, Professional Studies Review 2(1): 31-47. Quoted from pp. 44-45.

2 R. Lanham (1991), A Handlist of Rhetorical Terms, Berkeley, University of California Press. Quoted from pp. 102.

3 S. Newman (2002), Aristotle's Notion of "Bringing-Before-the-Eyes": Its Contributions to Aristotelian and Contemporary Conceptualizations of Metaphor, Style, and Audience, Rhetorica 20: 1-23.

4 E. Winsberg (2001), Simulations, Models, and Theories: Complex Physical Systems and their Representations, Philosophy of Science 8: S442-54. Quoted from p. S445.

5 E. Winsberg (1999), Simulation and the Philosophy of Science: Computationally Intensive Studies of Complex Physical Systems, Indiana University. Quoted from pp. 29-32.

6 E. Winsberg (1999); see 5, p. 29.

7 E. Winsberg (1999); see 5, p. 30.

8 E. Winsberg (1999); see 5, p. 32.

9 E. Winsberg (1999); see 5, p. 10.

10 E. Winsberg (1999); see 5, p. 32.

11 F. Pearce (2010, 9 February), Part two: How the 'climategate' scandal is bogus and based on climate sceptics' lies Environment. The Guardian. Retrieved May 13, 2010. Available at http://www.guardian.co.uk/environment/2010/feb/09/climategate-bogus-sceptics-lies.

12 J. Randerson (2010, 31 March), Climate researchers 'secrecy' criticised - but MPs say science remains intact. The Guardian. Retrieved May 13, 2010. Available at http://www.guardian.co.uk/environment/2010/mar/31/climatemails-inquiry-jones-cleared.

13 H.C. Foley, A.W. Scaroni and C.A. Yekel (2010, 3 February), RA-10 Inquiry Report: Concerning the Allegations of Research Misconduct Against Dr. Michael E. Mann, Department of Meteorology, College of Earth and Mineral Sciences, The Pennsylvania State University, The Pennsylvania State University. Retrieved May 14, 2010. Available at http://www.research.psu.edu/orp/Findings_Mann_Inquiry.pdf.

14 M. Herant, W. Benz, J. Hix, et al. (1994), Inside the Supernova: A Powerful Convective Engine, Astrophysical Journal 435: 339.

15 J. Blondin, A. Mezzacappa and C. DeMarino (2003), Stability of Standing Accretion Shocks, with an Eye Toward Core-Collapse Supernova, Astrophysical Journal 30 (584): 971-980.

16 J. Fahnestock (2002), Rhetorical Figures in Science, Oxford, Oxford University Press.

17 L. Ceccarelli (2001), Shaping Science with Rhetoric: The Cases of Dobzhansky, Schrodinger, and Wilson, Chicago, University of Chicago Press.

18 J. Bahcall (2004, April 28), Solving the Mystery of the Missing Neutrinos, Retrieved November 1, 2007. Available at http://nobelprize.org/nobel_prizes/physics/articles/bahcall/index.html.

19 B. Latour, S. Woolgar (1979), Laboratory Life: the Social Construction of Scientific Facts, Los Angeles: Sage.

20 D. Paul (2000), In Citing Chaos: A Study of the Rhetorical Use of Citations, Journal of Business and Technical Communication 14(2): 185-222.

21 C.S. Peirce (1997), Collected Papers of Charles S. Peirce Online, (InteLex Corporation) Retrieved March 8, 2004. Available at http://www.lib.utexas.edu. 
22 R. Lanham (1991); see 2, p. 54.

23 R. Lanham (1991); see 2, p. 38.

24 E. Winsberg (1999); see 5, p. 31.

25 R. Lanham (1991); see 2, p. 3.

26 R. Lanham (1991); see 2, p. 38.

27 L. Hughes Etzkorn (1997), A Metric-Based Approach to the Object-Oriented Reusable Software Components, Diss, University of Alabama.

28 L. Hughes Etzkorn (1997); see 27, p. 79.

29 G. Myers (1985), The Social Construction of Two Biologists' Proposals, Written Communication 2: 219-45.

30 G. Myers (1990), Writing Biology: Texts in the Social Construction of Scientific Knowledge, Madison, University of Wisconsin Press.

31 B. Latour (1999), Pandora's Hope: Essays on the Reality of Science Studies, Cambridge, Harvard University Press.

32 R. Harris (1997, March 25), Rhetoric of Science. Quoted from par. 8. Retrieved May 13, 2007. Available at http://web.archive.org/web/20070806150154/http:/www.ece.uwaterloo.ca/ jgwilkin/if/winter97/mar25/article.html.

33 P. Medarwar (1999), Is the Scientific Paper a Fraud? In E. Scanlon, R. Hill, \& K. Junker, Communicating Science: Professional Contexts (pp. 27-31), New York: Routledge.

34 J.M. Swales (1990), Genre Analysis, Cambridge, Cambridge University Press.

35 A. Gross (1996), The Rhetoric of Science. Cambridge: Harvard University Press.

36 A. Gross, J.E. Harmon (2002), Communicating Science: The Scientific Article from the 17th Century to the Present. New York, Oxford University Press.

37 C. Bazerman (1988), Shaping Written Knowledge: The Genre and Activity of the Experimental Article in Science, Madison, University of Wisconsin Press.

38 A. Galloway (2004), Protocol: How Control Exists after Decentralization, Cambridge: MIT Press.

39 E. Winsberg (2009), Computer Simulation and the Philosophy of Science, Philosophy Compass 4(5), 835-845. Quoted from p. 838.

40 H.J. Fell, V.K. Proulx and J. Casey (1996), Writing Across the Computer Science Curriculum, ACM SIGCSE Bulletin 28(1): 204-209.

41 A.B. Shiflet (2002), Computer Science with the Sciences: An Emphasis in Computational Science, SIGCSE Bulletin 34(4): 40-43.

42 J. Wolfe (2004), Why the Rhetoric of CS Programming Assignments Matters, Computer Science Education 14 (2): 147-63.

\section{Author}

Aimee Kendall Roundtree is an Associate Professor at the University of Houston-Downtown, where she teaches the rhetoric of science and medicine, hypermedia and technology, Web publishing and accessibility, visual design, and technical communication. Her research and publications examine scientific discursive practices, rhetorical implications of new media, visual design, and information technology. She is currently working on a book about the rhetorical nature of computer simulations and research projects examining new media in healthcare. E-mail: roundtreea@uhd.edu.

How TO CITE: A.K. Roundtree, The rhetoric of computer simulations in astrophysics: a case study, Jcom 09(03) (2010) A02. 